\title{
Hybrid key-Based Encryption in Cloud Storage
}

\author{
Su Min Shin1), Vandana Roy2)
}

\begin{abstract}
Hybrid key-based encryption (HKBE) is an open key cryptosystem and dispenses with the requests of open key framework (PKI) and authentication organization in traditional open key settings. Because of the nonattendance of PKI, the denial issue is a basic issue in IBE settings. A few revocable IBE plans have been proposed in regards to this issue. Recently, by implanting an outsourcing calculation procedure into IBE, Li et al. proposed a revocable IBE conspire with a key-redesign cloud specialist co-op (KU-CSP). Nonetheless, their plan has two inadequacies. One is that the calculation and correspondence expenses are higher than past revocable IBE plans. The other inadequacy is absence of versatility as in the KU-CSP must keep a mystery esteem for every client. In the article, we propose another revocable IBE plot with a cloud revocation authority (CRA) to understand the two deficiencies, in particular, the execution is altogether enhanced and the CRA holds just a framework mystery for every one of the clients. For security examination, we exhibit that the proposed plan is semantically secure under the decisional bilinear Diffie-Hellman (DBDH) supposition. At long last, we amplify the proposed revocable IBE plan to introduce a CRA-helped confirmation plot with period-restricted benefits for dealing with an expansive number of different cloud administrations.
\end{abstract}

Keywords : encryption, authentication, cloud computing, outsourcing computation, revocation authority.

\section{Introduction}

Character (ID)-based open key system (ID-PKS) [1-2] is an appealing option for open key cryptography. ID-PKS setting dispenses with the requests of open key foundation (PKI) and endorsement organization in ordinary open key settings. An ID-PKS setting comprises of clients and a trusted outsider (i.e. private key generator, PKG). The PKG is mindful to produce every client's private key by utilizing the related ID data (e.g. email address, name or government disability number). Accordingly, no authentication and PKI are required in the related cryptographic systems under ID-PKS settings. In such a case, ID-based encryption (IBE) permits

Received(May 16, 2016), Review Result(1st: May 31, 2016, 2nd: June 24, 2016), Accepted(September 10, 2016)

${ }^{1}$ Chung-Ang University, \#301-116, 221, Huksok-dong, DongJak-Gu Seoul, Rep. of Korea email: sinsumin72@gmail.com

${ }^{2}$ (Corresponding Author) Department of Computer Science and Engineering, KL University email: vandanaroy@gmail.com 
a sender to scramble message straightforwardly by utilizing a collector's ID without checking the approval of open key authentication. In like manner, the recipient utilizes the private key related with her/his ID to unscramble such ciphertext. Since an open key setting needs to give a client repudiation component, the exploration issue on the most proficient method to deny getting out of hand/bargained clients in an ID-PKS setting is normally raised. In traditional open key settings, endorsement disavowal list (CRL) [3] is an outstanding denial approach. In the CRL approach, if a gathering gets an open key and its related authentication, she/he first approves them and afterward turns upward the CRL to guarantee that the general population key has not been disavowed. In such a case, the methodology requires the online help under PKI with the goal that it will bring about correspondence bottleneck. To enhance the execution, a few effective renouncement instruments [4-8] for traditional open key settings have been all around examined for PKI. Without a doubt, specialists likewise focus on the repudiation issue of ID-PKS settings. A few revocable IBE plans have been proposed with respect to the repudiation instruments in ID-PKS settings.

\section{Proposed system}

\subsection{Related Work}

In 2001, Boneh and Franklin [2] proposed the primary useful IBE conspire from the Weil matching and recommended a straightforward denial technique in which each non-disavowed client gets another private key produced by the PKG occasionally. A period can be set as a day, seven days, a month, and so on. A sender uses an assigned recipient's ID and current period to encode messages while the assigned beneficiary decodes the ciphertext utilizing the present private key. Henceforth, it is vital for the clients to upgrade new private keys intermittently. To renounce a client, the PKG just quits giving the new private key to the client. Clearly a safe channel must be built up between the PKG and every client to transmit the new private key and this would bring about overwhelming burden for the PKG.

With a specific end goal to ease the heap of the PKG in Boneh and Franklin's plan, Boneh et al. [9] proposed another denial technique, called prompt repudiation. Prompt renouncement strategy utilizes an assigned semi-trusted and online specialist (i.e. arbiter) to relieve the administration heap of the PKG and help clients to unscramble ciphertext [10-13]. In such a case, the online middle person must hold shares of the considerable number of clients' private keys. Since the unscrambling operation must include both sides, neither the client nor the 
online go between can cheat each other[14-18]. At the point when a client was disavowed, the online middle person is told to quit helping the client. In any case, the online go between must help clients to unscramble each ciphertext with the goal that it turns into a bottleneck for such plans as the quantity of clients develops massively.

\subsection{Existing System}

Li et al.[19] brought an outsourcing calculation system into IBE to propose a revocable IBE plot with a key-update cloud specialist organization (KU-CSP). They moves the key-overhaul methods to a KU-CSP to ease the heap of PKG.

Li et al. [19] additionally utilized the comparable method received in Tseng and Tsai's plan, which segments a client's private key into a personality key and a period redesign key. The PKG sends a client the comparing character key by means of a protected channel. Then, the PKG must create an arbitrary mystery esteem (time key) for every client and send it to the KU-CSP.

Then the KUCSP produces the present time upgrade key of a client by utilizing the related time key and sends it to the client through an open channel.

\subsubsection{Disadvantage of Existing System}

ID-based encryption (IBE) permits a sender to encrypt message specifically by utilizing a recipient's ID without checking the approval of open key certificate[20-22].

In existing system misbehaving/compromised users in an ID-PKS setting is normally raised. Immediate denial strategy utilizes an assigned semi-trusted and online authority (i.e. mediator) to alleviate the administration heap of the PKG and help clients to decrypt ciphertext.

The computation and communication costs are higher than past revocable IBE schemes. The other inadequacy is un-adaptability as in the KU-CSP must keep a period key for every client so it will incur the management load.

\subsection{Proposed System}

In request to tackle both the un-adaptability and the inefficiency in $\mathrm{Li}$ et al's. [19] scheme, we propose another revocable IBE scheme with cloud revocation authority (CRA).

In specific, every client's private key still comprises of a personality key and a period 
overhaul key. We present a cloud repudiation expert (CRA) to supplant the part of the KU-CSP in Li et al's. [19] plan. The CRA just needs to hold an arbitrary mystery esteem (ace time key) for every one of the clients without influencing the security of revocable IBE conspire.

The CRA utilizes the ace time key to produce the present time redesign key occasionally for each non-revoked client and sends it to the client by means of an open channel. It is apparent that our plan takes care of the un-versatility issue of the KU-CSP.

We build a CRA-helped verification conspire with period-constrained benefits for dealing with an expansive number of different cloud administrations.

\subsubsection{Advantages of Proposed System}

The proposed scheme has the benefits of both Tsengand Tsai's revocable IBE plan and Li et al's. [19] plan.

The proposed exhibit the structure of our revocable IBE scheme with CRA and characterize its security ideas to model conceivable threats and attacks CRA-aided confirmation scheme with period-limited benefits for dealing with an expansive number of different cloud administrations.

\section{Conclusion}

In this article, we proposed another revocable IBE conspire with a cloud denial expert (CRA), in which the disavowal methodology is performed by the CRA to reduce the heap of the PKG. This outsourcing calculation method with different experts has been utilized in Li et al's. revocable IBE conspire with KU-CSP. Nonetheless, their plan requires higher computational and communicational expenses than beforehand proposed IBE plans. For the time key overhaul technique, the KU-CSP in Li et al's. plan must keep a mystery esteem for every client with the goal that it is absence of adaptability. In our revocable IBE conspire with CRA, the CRA holds just an ace time key to play out the time key redesign systems for every one of the clients without influencing security. As contrasted and $\mathrm{Li}$ et al's. [19] plan, the exhibitions of calculation and correspondence are fundamentally made strides. By exploratory outcomes and execution investigation, our plan is appropriate for cell phones. For security examination, we have exhibited that our plan is semantically secure against versatile ID assaults under the decisional bilinear Diffie-Hellman suspicion. At last, in view of the proposed revocable IBE conspire with CRA, we developed a CRA aided validation plot with period-restricted benefits 
for dealing with an extensive number of different cloud administrations.

\section{References}

[1] A. Shamir, Identity-based cryptosystems and signature schemes Proc. Crypto'84, LNCS, (1984), Vol.196, pp.47-53.

[2] D. Boneh and M. Franklin, Identity-based encryption from the Weil pairing, Proc. Crypto'01, LNCS, (2011), Vol.2139, pp.213-229.

[3] R. Housley, W. Polk, W. Ford, and D. Solo, Internet X.509 Public Key Infrastructure Certificate and Certificate Revocation List (CRL) Profile, IETF, RFC 3280, (2002).

[4] W. Aiello, S. Lodha, and R. Ostrovsky, Fast digital identity revocation, Proc. Crypto'98, LNCS, (1998), Vol.1462, pp.137-152.

[5] M. Naor and K. Nissim, Certificate revocation and certificate update, IEEE Journal on Selected Areas in Communications, (2000), Vol.18, No.4, pp.561-570.

[6] S. Micali, Novomodo: Scalable certificate validation and simplified PKI management, Proc. 1st Annual PKI Research Workshop, (2002), pp.15-25.

[7] F. F. Elwailly, C. Gentry, and Z. Ramzan, QuasiModo: Efficient certificate validation and revocation Proc. PKC'04, LNCS, (2004), Vol.2947, pp.375-388.

[8] V. Goyal, Certificate revocation using fine grained certificate space partitioning, Proc. Financial Cryptography, LNCS, (2007), Vol.4886, pp.247-259.

[9] D. Boneh, X. Ding, G. Tsudik, and C.-M. Wong, A Method for fast revocation of public key certificates and security capabilities Proc.10th USENIX Security Symp., (2001), pp.297-310.

[10] X. Ding and G. Tsudik, Simple identity-based cryptography with mediated RSA, Proc. CT-RSA'03, LNCS, (2003), Vol.2612, pp.193-210.

[11] B. Libert and J. J. Quisquater, Efficient revocation and threshold pairing based cryptosystems, Proc. PODC2003, (2003), pp.163-171.

[12] J. Baek and Y. Zheng, Identity-based threshold decryption, Proc. PKC'04, LNCS, (2004), Vol.2947, pp.262-276.

[13] H. S. Ju, D. Y. Kim, D. H. Lee, H. Park, and K. Chun, Modified ID-based threshold decryption and its application to mediated ID based encryption Proc. APWeb2006, LNCS, (2006), Vol.3841, pp.720-725.

[14] A. Boldyreva, V. Goyal, and V. Kumar, Identity-based encryption with efficient revocation, Proc. CCS'08, (2008), pp.417-426.

[15] A. Sahai and B. Waters, Fuzzy identity-based encryption, Proc. Eurocrypt'05, LNCS, (2005), Vol.3494, pp.557-557.

[16] B. Libert and D. Vergnaud, Adaptive-ID secure revocable identity-based encryption, Proc. CT-RSA'09, LNCS, (2009), Vol.5473, pp.1-15. 
[17] J. H. Seo and K. Emura, Revocable identity-based encryption revisited: security model and construction, Proc. PKC'13, LNCS, (2013), Vol.7778, pp.216-234.

[18] S. Park, K. Lee, and D. H. Lee, New constructions of revocable identity-based encryption from multilinear maps, IEEE Transactionson Information Forensics and Security, (2015), Vol.10, No.8, pp.1564-1577.

[19] C. Wang, Y. Li, X. Xia, and K. Zheng, An efficient and provable secure revocable identity-based encryption scheme, PLoS ONE, (2014), Vol.9, No.9, article: e106925.

[20] A. Lewko A and B. Waters, New techniques for dual system encryption and fully secure hibe with short ciphertexts Proc.TCC'10, LNCS, (2010), Vol.5978, pp.455-479.

[21] J. H. Seo and K. Emura, Efficient delegation of key generation and revocation functionalities in identity-based encryption, Proc. CT-RSA'13, LNCS, (2013), Vol.7779, pp.343-358.

[22] J. H. Seo and K. Emura, Revocable hierarchical identity-based encryption: history-free update, security against insiders, and short Ciphertexts, Proc. CT-RSA'15, LNCS, (2015), Vol.9048, pp.106-123. 